\title{
La crianza positiva de los niños y niñas. Una mirada desde el ordenamiento jurídico cubano
}

\section{The Positive Uupbringing of Boys and Girls. A Look from the Cuban Legal System}

Yusmely Soto López.* https://orcid.org/0000-0003-1004-208X http://dx.doi.org/10.21503/lex.v18i26.2197

* Licenciada en Derecho-Profesora Asistente del Departamento de Derecho de la Universidad de Granma, Cuba.

Correo electrónico: ysotol@udg.co.cu

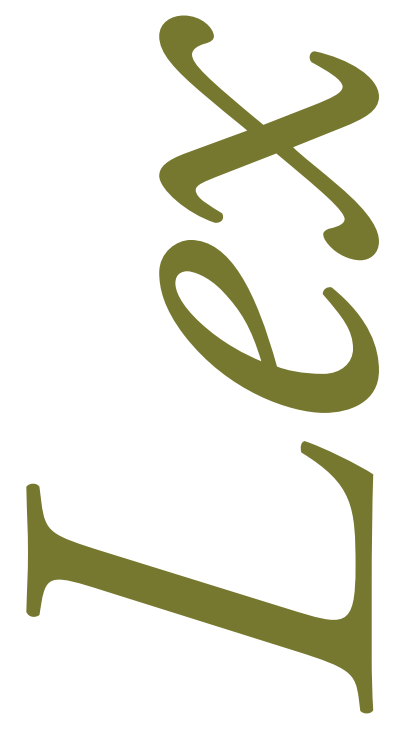

(c) (C) Los autores. Artículo publicado por la Revista Lex de la Facultad de Derecho y Ciencias Políticas de la Universidad Alas Peruanas. Este es un artículo de acceso abierto, distribuido bajo los términos de la Licencia Creative Commons Atribución-No Comercial-Compartir Igual 4.0 Internacional.(http://creativecommons.org/licenses/by-nc-sa/4.0/), que permite el uso no comercial, distribución y reproducción en cualquier medio, siempre que la obra original sea debidamente citada. 


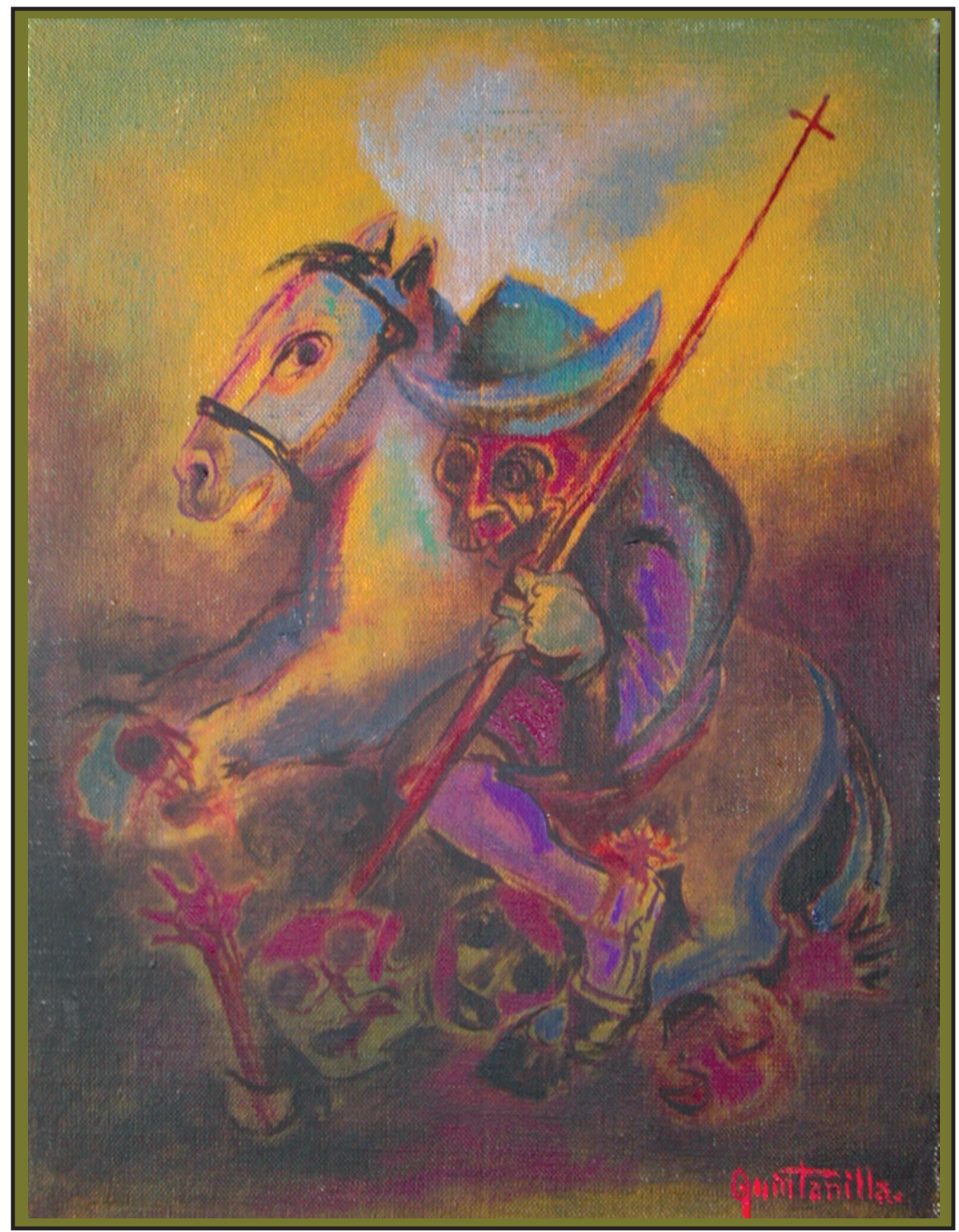

Santiago mata indios. Óleo, 1999. Artista plástico peruano, Alberto Quintanilla, (Cusco, 1934). 


\section{RESUMEN}

La crianza positiva de los infantes es un tema multidisciplinario que no deja de vincular a las ciencias jurídicas, y dentro de esta a la Disciplina de Derecho Civil y de Familia. Dada la vulnerabilidad social y económica que caracteriza a la niñez, sus derechos se constituyen en responsabilidad de la familia; pero a su vez es responsabilidad del Estado y la sociedad crear y consolidar condiciones que permitan a las familias el fiel cumplimiento de los derechos de la población infantil. El objetivo del presente trabajo está orientado a fundamentar la necesidad de una crianza positiva en los niños y niñas a partir de su concepción teórica doctrinal y su expresión en el Ordenamiento Jurídico cubano; en vistas al perfeccionamiento del reconocimiento y observancia de los derechos de la población infantil en Cuba.

Palabras claves: crianza positiva, educación de niños y niñas, violencia de género.

\section{ABSTRACT}

The positive upbringing of infants is a multidisciplinary subject that does not stop linking the legal sciences, and within this to the Discipline of Civil and Family Law. Given the social and economic vulnerability that characterizes children, their rights are the responsibility of the family; but in turn it is the responsibility of the State and society to create and consolidate conditions that allow families to faithfully fulfill the rights of the child population. The objective of this work is aimed at establishing the need for positive upbringing in boys and girls based on their theoretical doctrinal conception and their expression in the Cuban Legal System; with a view to improving the recognition and observance of the rights of the child population in Cuba.

key words: positive parenting, education of boys and girls, gender violence. 


\section{INTRODUCCIÓN}

La obligación que se tiene con la educación de los niños y de las niñas y de las personas adolecentes lleva a la necesidad de enriquecer su formación de la mejor manera posible para lo cual es importante promover una crianza libre de violencia, enmarcada dentro de un enfoque de derechos, sin discriminaciones y con equidad de género.

La profesora catedrática de la Universidad de Costa Rica, Carballo Sonia, fundamenta que la condición propia de los infantes como personas en pleno crecimiento que dependen de personas adultas significativas como son sus madres, padres, abuelos y demás cuidadores; obliga a analizar los múltiples retos que su crianza involucra. ${ }^{1}$

Para estos seres humanos educarlos implica, de parte de las personas adultas significativas, un manejo de límites que permita una estimulación adecuada y oportuna para su desarrollo físico, emocional, cognoscitivo y social. Cada una de estas dimensiones subraya un aspecto particular del desarrollo y entre ellas hay una interdependencia considerable, por lo que cada dimensión refleja a las otras. El desarrollo humano se ha convertido en una ciencia multidisciplinaria que toma conocimientos actualizados de la biología, la fisiología, la medicina, la educación, la psicología, la sociología y la antropología.

Aunque Cuba cuenta con una Sistema legislativo de avanzada para la protección de la niñez y la adolescencia, esto no conduce de forma automática y espontánea a su cumplimiento pleno, subsistiendo prácticas de crianza violadoras de estos derechos donde el castigo físico se toma como medida correctiva para corregirlos y corregirlas.

Se exponen a continuación fundamentos teóricos que acentúan la importancia de educar a los niños y niñas como personas de derechos, en la familia, en la escuela y en la comunidad en general, así como su expresión normativa en el Ordenamiento Jurídico cubano, teniendo en cuenta la responsabilidad del Estado y la sociedad de crear y consolidar condiciones que permitan a las familias el fiel cumplimiento de los derechos de la población infantil.

1. Sonia Carballo Vargas, "Desarrollo Humano y Aprendizaje: Practicas de crianza de las madres Jefas de Hogar", Revista Electrónica, Actualidades Investigativas en la Educación. Universidad de Costa Rica 6, 2 (2006): 2. 


\section{FUNDAMENTOS TEÓRICOS DOCTRINALES DE UNA CRIANZA POSITIVA DE LOS NIÑOS Y NIÑAS}

\section{. Que significa crianza positiva?}

«Tienes que hacerlo porque lo digo yo, y no se hable más». Y el niño no sabía si llorar o acatar con obediencia la orden de su papá, que no entendió la poca habilidad de su hijo para amarrarse los cordones de sus zapatos. «No me digas que no sabes que ya te he enseñado muchas veces, y cuando termines, acuéstate. Hoy no hay muñequitos ni aventuras», y al infante no le extrañó, pues el día anterior tampoco vio la televisión por demorarse mientras comía.

«Óigame, mamá, no es fácil lidiar con su hijo. Todo lo cuestiona, quiere saber el porqué de todo lo que oriento en el aula, y dice que en su casa le explican siempre antes de pedirle que haga caso. ¿Ustedes están criando bien a su hijo? Se cree con el derecho de entenderlo todo, y hay cosas que deben hacerse según lo establecido, y ya».

¿Cómo debemos criar a nuestros hijos? ¿Cuál es el estilo de autoridad que debe primar en el hogar para lograr que, al paso del tiempo, el niño se convierta en un adulto educado, autónomo, capaz de convivir y participar en sociedad? ¿Acaso los padres saben siempre cuál es el mejor camino?

Por lo primordial y sensible de este tema innumerables son las definiciones que explican y enfocan la crianza positiva de los niños y las niñas (seres humanos menores de 18 años), pero la mayoría de ellas coinciden en los principales aspectos que la conforman.

Para la profesora Carballo $S$, "una práctica de crianza positiva es permitir que los niños se relacionen con personas, plantas y cosas del mundo que les rodea para que su pensamiento lógico se desarrolle al procesar y utilizar información”. ${ }^{2}$

Para la Maestra y Psicopedagoga María José Roldán, "la crianza positiva fomenta la relación entre padres e hijos basada en el respeto mutuo, ayuda a que los pequeños se desarrollen de forma adecuada, y que además los hijos se críen para que sepan relacionarse con los demás de forma no violenta y constructiva. Donde los padres comparten una visión común: utilizar el cariño como base de la educación" ${ }^{3}$

Para el Instituto Colombiano de bienestar familiar, "la crianza positiva busca promover un estilo de educación sin violencia, disciplina con amor y vínculos afectivos fuertes y afianzar diferentes formas para relacionarse con los nińos(as) desde el reconocimiento y el afecto. En la crianza positiva ambos padres, cuidadores y agentes educativos tienen expectativas reales de lo que pasa con los niños, entendiendo que hay cosas que ellos son capaces de controlar y otras que no, que hay momentos en que los

2. Ibídem, 17.

3. María José Roldán, “La crianza positiva de los hijos - Guía infantil”, acceso el 22 de enero 2019, https://www.guiainfantil.com 
niños hacen travesuras, que no es por maldad, sino que hacen parte de su proceso de desarrollo normal y de exploración del espacio físico en el que viven" ${ }^{4}$

Otro criterio consiste en que "la crianza positiva es educar a los hijos, siempre desde el respeto y el carińo incondicional hacia ellos, donde no falten normas y limites coherentes para que entiendan, que el mundo viene con normas y los castigos no deben estar presentes porque no son necesarios para criar niños felices. Cómo llevar a cabo una crianza positiva". ${ }^{5}$

Consideramos entonces sistematizando todas estas razones, que cuando educamos a través de la crianza positiva, fomentamos en los niños y niñas las habilidades, capacidades y actitudes necesarias para la vida, abrimos nuestra mente a nuevas y beneficiosas formas de crianza, fragmentamos las creencias de que educar en el autoritarismo es lo mejor, damos al niño libertad de expresión. Les permitimos crecer en un hogar donde existe el amor, el diálogo abierto y el respeto, donde no tienen cabida ni los golpes ni los castigos físicos.

\section{. Modelos de crianza}

Entre los modelos de crianza que podemos asumir con nuestros hijos se encuentran el autoritario, el permisivo y el democrático, y cada uno de estos permitirá formar un individuo con determinadas características en el plano individual y social. ${ }^{6}$

Muchas generaciones crecieron al amparo de una familia autoritaria, donde la dura verticalidad de los progenitores conlleva a la obediencia sin criterios, la que en algunas situaciones puede desencadenar absoluta sumisión o rebeldía. Esta manera de educar utiliza, en no pocas ocasiones, el maltrato físico y los castigos; impide la comunicación con los hijos porque el respeto a las normas se gana con imposición. Son familias en las que prima el control, pero falta el amor, o en las que no se sabe cómo demostrarlo sin perder el rol del que manda.

No pocas familias en Cuba, al igual que muchas de los niveles medio y alto de otras sociedades, adoptan el estilo de crianza permisivo, y culpan por ello al período especial. Las carencias materiales que sufrimos en esa etapa sirven de justificación para quienes, como padres, quieren que no les falte nada a sus hijos y que no les abrumen los regaños ni las reglas. La permisividad toca extremos peligrosos, pues en estos hogares no existen reglas para los pequeños.

En familias permisivas se forman individuos con bajo nivel de responsabilidad, caprichosos y exigentes para hacer siempre su voluntad. Pueden ser inadaptados en cualquier ámbito, aunque se puede

4. Cartilla 8 "Crianza Positiva" del Instituto Colombiano de bienestar familiar-ICBF (2015): 2-3.

5. "Como llevar a cabo una crianza positiva" acceso el 2 de octubre de 2019, https://www.bekiapadres.com/articulos/como-llevar-cabo-crianza-positiva/

6. Ana María Domínguez Cruz, "Como ser mejores madres y padres", Juventud Rebelde, martes 29 de Septiembre de 2015. 
lograr su inserción y entendimiento a través del trato afectivo, que no les faltó en el hogar.

Un elevado nivel de comunicación y participación en el hogar, así como la aceptación y respeto de las diferencias son elementos positivos que se generan en las familias que siguen el método de crianza democrático, que no por ser el más aconsejable es el más fácil de mantener. Predomina la horizontalidad en las relaciones entre padres e hijos sin que los primeros pierdan su rol de decisores y responsables de la autoridad. Se maneja la persuasión, el diálogo, y se fomenta el entendimiento a partir de la explicación llana y profunda.

Los niños entienden el porqué de lo que deben y pueden hacer, y sus criterios son tomados en cuenta, sin que trascienda a asuntos que no le competen por su edad. Al crecer, serán individuos capaces de usar los espacios de participación social y defender sus criterios, sabrán elegir y tomar decisiones, y no acatarán pasivamente lo que no pueden comprender. Se corren riesgos con el modelo democrático, y los padres debemos estar conscientes de ello. En la búsqueda de un diálogo efectivo podemos perder los límites, y hay que percatarse de que hay diferentes maneras de aplicación del método en dependencia de la gravedad de la situación, pues no se conversa y se convence igual a un niño que deja de hacer la tarea que a uno que tomó lo que no es suyo en el aula y lo trajo para la casa.

\section{. Como llevar a cabo una crianza positiva?}

Hay padres que se escudan en que la crianza que han recibido ellos por parte de sus padres a pesar de haber sido autoritaria u otro estilo de crianza, no ha tenido problemas en su educación y que la crianza positiva es sólo una moda. Muchos otros padres al ver la eficacia de este estilo de crianza en la educación de los niños, han empezado a interesarse más en este tema, para poder aprender y criar a sus hijos como felices y no sometidos. Un niño feliz se convertirá en un adulto con éxito, en cambio un niño sometido se convertirá en un adulto sometido y probablemente con problemas emocionales graves. ${ }^{7}$

Pero la realidad es que muchos progenitores intentan hacer las cosas lo mejor que pueden o que saben, y en muchos casos es falta de información que los padres no sepan aplicar algunas estrategias educativas adecuadas, cuando no se sabe que estilo de crianza seguir, los padres se pueden sentir perdidos. Es el adulto el que debe entender al niño(a), es importante explicarles porque hay cosas que no deben hacer, siempre manteniendo un dialogo, escuchando y respondiendo sus inquietudes. También es importante que los adultos reconozcan sus propias emociones como a rabia, la frustración, el miedo, que pueden en una situación adversa llevar a castigos corporales y humillantes. Cartilla "Crianza Positiva (2015)" 8 .

Para lograr una crianza positiva, y que los niños crezcan felices y los padres sin sentimiento de culpabilidad porque creen que lo están haciendo bien, se deben seguir las siguientes estrategias:

7. "Como llevar una crianza positiva”, op. cit.

8. Cartilla 8 "Crianza Positiva" del ICBF, op. cit., 3, vid. Nota 1. 
Los padres deben comprometerse a regular sus propias emociones.

Los padres dan prioridad a mantener y reforzar la conexión y el vínculo emocional entre padres e hijos.

Los padres deben amar a sus hijos incondicionalmente. Para ello deberán olvidar las técnicas de castigo o recompensas que se utilizan para controlar o manipular a los niños. Los padres deberán sentirse como entrenadores que ofrecen a los peques una guía amorosa para que estos sean capaces de manejar sus emociones y comportamiento.

El castigo es destructivo para la relación con tu hijo, además que afecta muy negativamente su desarrollo emocional y social. Siempre que tengas que poner límites es mejor hacerlo de un modo empático para que el niño se dé cuenta de que te importa lo que siente, así podrá mejorar su comportamiento y al mismo tiempo, no estará enfadado contigo (aunque estés intentando hacer las cosas bien).

El desafío siempre será un problema de relación, si tu hijo no acepta lo que dices, es un indicador de que la relación no es lo suficientemente fuerte como para apoyar la enseñanza de lo que le estas intentando inculcar. La lucha de poder solo hará que la brecha emocional entre vosotros sea demasiado grande. ${ }^{9}$

Con la crianza positiva ayudas a tus hijos a sentirse personas importantes y valiosas, lo que traerá como consecuencia que sean desde niños seres felices, maduros, respetuosos, con habilidades y capacidades para resolver conflictos, seres humanos colaboradores y responsables.

\section{. Los niños y niñas víctimas de la violencia de género}

La autora española Horno Goicoechea Pepa, en investigaciones realizadas en el país Ibérico en siete comunidades autónomas explica que los pequeños también son víctimas directas de la violencia de género que se ejerce hacia sus madres, no solo porque se les agrede a ellos físicamente, sino porque siempre son víctimas de violencia psicológica cuando presencian las palizas o las agresiones a sus madres y porque por el mero hecho de vivir en un entorno donde la violencia es una pauta de relación. Ven y sufren una madre maltratada en vez de protectora; ven y sufren a un padre maltratador en vez de protector. ${ }^{10}$

Entre los efectos de la violencia de género sobre niños(as) identificados por Save The Children (Organización que trabaja en la defensa y promoción de los derechos del niño) y los profesionales entrevistados, se destacan los siguientes:

9. "Como llevar a cabo una crianza positiva" acceso 16 de marzo de 2019, https://www.bekiapadres.com/articulos/como-llevar-cabo-crianza-positiva/

10. Pepa Horno Goicoechea, “Atención a los niños y las niñas víctimas de la violencia de género". Revista Intervención Psicosocial, 15, 3 (2006): 307-316. 
- Problemas de socialización: dificultad para establecer relaciones personales, conductas agresivas o auto agresivas.

- Problemas escolares: bajo rendimiento académico concentración.

- Síntomas de estrés postraumático: trastorno de sueño, fobias, ansiedad, ataque de pánico, entre otros.

- Alteraciones de desarrollo afectivo: modelos de vinculación erróneos, en los que violencia y amor van unidos.

El sistema de protección español no contempla a los nińos como víctimas de la violencia de género lo que posibilita su victimización secundaria. Los menores son tratados como objeto de protección, no como sujetos de derecho. No se les escucha a los infantes en el proceso judicial.

\section{. La crianza positiva de los niños y niñas. Una mirada desde el Ordenamiento Jurídico cubano}

En Cuba como en otras latitudes la célula básica de la sociedad se ha diversificado, y es válido reconocer que, por consiguiente, los estilos de crianza se han transformado y en no pocos casos coexisten algunos en la misma organización familiar.

Desde el triunfo de la Revolución los niños y las niñas han sido protegidos y tutelados por el Estado cubano, potenciado su sano e íntegro desarrollo a través de los diferentes programas sociales, educacionales y de salud ejecutados. La superestructura jurídica tampoco escapa a esta realidad.

En el sistema de disposiciones jurídicas que protege a la nińez y a los adolescentes tenemos que: Cuba desde enero de 1990, es signataria de la Convención de los Derechos del Niño ${ }^{11}$, la ratificó el 21 de agosto de 1991, y entró en vigor un mes después, el 20 de septiembre, cuando fue publicado su texto íntegramente en la Gaceta Oficial, con una declaración firmada por el Presidente del Consejo de Estado, para así quedar refrendados en un cuerpo legal los derechos de la nińez y la adolescencia. Es de carácter obligatorio para los países firmantes adoptar las medidas necesarias para dar efectividad a todos los derechos reconocidos en la Convención e informar al Comité de los Derechos del niño sobre los pasos que han adoptado para aplicar lo establecido. Este valioso instrumento a lo largo de sus 54 artículos, reconoce que los niños son individuos con derecho de pleno desarrollo físico, mental y social, y con derecho a expresar libremente sus opiniones, se rige por cuatro principios que son la guía suprema de cada uno de los artículos de este Tratado Internacional de los Derechos Humanos y son la base para que los derechos del niño(a) se conviertan en realidad:

11. "Principios rectores de la Convención sobre los Derechos del Niño: una explicación fácil", acceso 3 de abril de 2019, https://www.crin.org/es/biblioteca/publicaciones/principios-rectores-de-la-convencion-sobre-los-derechos-delnino-una 
1. Principio de "No discriminación" (Artículo 2). No hay causa que justifique el trato desigual de los niños cualquiera que sea su raza, religión y habilidades, idioma, discapacidad o familia de la que proviene.

2. Principio de observar siempre el interés superior del niño ${ }^{12}$ (artículo 3). Los adultos deben tomar decisiones y hacer aquello que es beneficioso para los niños. Este principio se manifiesta por ejemplo en la creación de nuevas leyes, políticas del gobierno y presupuesto destinado a la niñez.

3. Principio del derecho a la vida, la supervivencia y el desarrollo (artículo 6).

4. Principio de participación y ser escuchado (Articulo 12). Los padres deben escuchar las opiniones de los hijos e involucrarlos en la toma de decisiones, reconoce que el nivel de participación en las decisiones debe de ser apropiado para su nivel de madurez.

La nueva Constitución de la República de Cuba en el Título V consagra el Capítulo III a "Las Familias" en sus artículos 84,85 y 86 reconoce a los niños, niñas y adolescentes como plenos sujetos de derechos y gozan de aquellos reconocidos en esta Constitución, además de los propios de su especial condición de persona en desarrollo. Son protegidos contra todo tipo de violencia.

Establece la obligación no solo de madres, padres en su guarda y cuidado, sino también de otros parientes consanguíneos o afines que cumplan estas funciones, los que tienen el deber de brindarles alimentos, respetar y garantizar el pleno ejercicio de sus derechos y protegerlos de todo tipo de violencia y contribuir activamente al desarrollo pleno de su personalidad.

Así mismo la responsabilidad del Estado, la sociedad y la familia en garantizar que estos tengan un desarrollo armónico e integral para lo cual tienen en cuenta su interés superior en las decisiones y actos que le conciernan.

El Código de la Niñez y la Juventud de Cuba, Ley No. 16 de 28 de junio de 1978, establece normas especiales reguladoras de los derechos de los niños y jóvenes

En su articulado refiere los valores que deben ser inculcados a las nuevas generaciones, como son: el amor a la patria y al estudio, el hábito de practicar deportes, y principios como el de internacionalismo y la igualdad. Hace referencia al papel protagónico de la familia en la formación moral, física y espiritual de sus miembros más jóvenes; reconoce el papel del Estado en su formación, garantizando los medios necesarios para el disfrute de sus derechos y la capacitación del personal encargado de ello.

12. Todas las medidas respecto del niño deben estar basadas en la consideración del interés superior del mismo. Corresponde al Estado asegurar una adecuada protección y cuidado, cuando los padres y madres, u otras personas responsables, no tienen capacidad para hacerlo. 
Resalta el papel de la escuela en la educación de niños y jóvenes; y el planteamiento de que el Estado presta especial atención a las escuelas para niños discapacitados física o mentalmente, así como aquellas que se encargaran de la educación de los niños con trastornos de conducta respectivamente ${ }^{13}$.

El artículo 45 de la Ley del Registro del Estado Civil contempla que corresponderá como primer apellido el primero del padre y como segundo, el primero de la madre. Esta Ley asegura además, el derecho de los nińos a ser reconocidos por sus padres y establece procedimientos que garantizan que todos puedan quedar debidamente inscriptos inmediatamente después de su nacimiento, sin diferenciarlos por el estado conyugal de sus padres.

El Decreto Ley No. 64 de 1982 "Sobre el Sistema de Atención a Menores con trastornos de Conducta”, excluye de la jurisdicción penal a los menores de 16 años transgresores de las normas penales, los que son atendidos a través de un procedimiento administrativo en el que intervienen especialistas y funcionarios de los Ministerios del Interior y de Educación fundamentalmente ${ }^{14}$.

El Decreto Ley No. 76 de 1984 crea la Red Nacional de Círculos Infantiles Mixtos y Hogares de Menores sin Amparo Familiar, para la protección de los niños en desventaja social.

La Ley No. 62 de 1987. Código Penal contiene figuras delictivas que protegen a los menores de edad contra el maltrato, el abandono, los delitos sexuales, etc. Con independencia de medidas civiles que se pueden tomar, como retirarles la patria potestad a los padres que incurran en estos hechos.

El Código de Familia. Ley No 1289 de 1975 es expresión de la importancia que nuestra sociedad le confiere a la familia y establece el rol protagónico que le corresponde en la formación de los hijos y reafirma el principio de igualdad de los hijos, así como la obligación de los padres de protegerlos y de atender su alimentación, salud y educación.

No obstante, son muchos los retos que debe enfrentar la modificación del actual Código de Familia, en consonancia con la nueva Constitución, los lineamientos de las Convenciones de Naciones Unidas sobre la eliminación de todas las formas de discriminación contra la Mujer y sobre los Derechos del niño.

La esfera de Trabajo Comunitario de la Federación de Mujeres Cubanas, de conjunto con la Sociedad Cubana de Derecho Civil y de Familia, de la Unión Nacional de Juristas de Cuba ha trabajado en la elaboración de un anteproyecto de Código de Familia ${ }^{15}$. La nueva Ley debe establecer expresamente los

13. Vid. El Código de la niñez y la Juventud. Ley No.16 de 28 de junio de 1978 en sus artículos 3, 4, 5, 20 y 29.

14. "Sistema de leyes que protegen la niñez y a los adolescentes en Cuba", acceso 13 de febrero de 2019, http://civica.cubaeduca.cu/sistema-de-leyes-que-protegen-a-la-ninez-y-a-los-adolescentes-en-cuba\#: :text=\%C2\%B FConoces\%20cu\%C3\%A11\%20es\%20el\%20sistema,los\%20adolescentes\%20en\%20nuestro\%20pa\%C3\%ADs\%3F\& text=En\%20el\%20sistema\%20de\%20disposiciones,regulado\%20en\%20la\%20Ley\%20No.

15. Dora Pérez Sáez, "Nuevo Código de Familia favorece a los niños", Periódico Juventud Rebelde, Diario de la Juventud Cubana, de 23 de marzo de 2019. 
derechos que tendrían los niños sujetos al régimen de patria potestad, aspecto no contenido de manera explícita en la Ley vigente, y entre los que pudiéramos mencionar:

- $\quad$ el derecho a ser protegido de toda forma de violencia física y psíquica, explotación, abuso sexual, descuido o trato negligente.

- $\quad$ el derecho de los infantes a participar activamente en la vida familiar y social, a que sus opiniones sean escuchadas, a defender sus criterios y a recibir argumentos que puedan convencerlos.

- moderar el derecho de los padres, ya permitido por la Ley vigente, de corregir a sus hijos, pero esclareciendo que dicha corrección debe ser adecuada, moderada, sin el empleo de violencia física o psíquica, ni con métodos humillantes.

- la remisión a la Ley penal para los casos de padres que incumplan los deberes inherentes a la patria potestad, o aquellos que se excedan en la corrección sobre sus hijos. ${ }^{16}$

Con la nueva norma también se ayudaría a los abuelos. A menudo vemos que, si los padres van a trabajar al exterior, generalmente aquellos quedan a cargo del niño. Sin embargo, no tienen amparo legal para realizar ninguna acción con los nietos. Si les otorgamos el régimen de guarda y cuidado a esos abuelos, les facilitaríamos las acciones legales. Incluso, hay padres que abandonan a sus hijos, que no se ocupan más de ellos. En esos casos, ¿̨por qué no deferir la patria potestad a favor de los abuelos?

\section{CONCLUSIONES}

La crianza positiva busca promover un estilo de educación sin violencia, disciplina con amor y vínculos afectivos fuertes, a través de esta ayuda a tus hijos a sentirse personas importantes y valiosas, lo que traerá como consecuencia que sean desde niños seres felices, maduros, respetuosos, con habilidades y capacidades para resolver conflictos, seres humanos colaboradores y responsables.

Entre los modelos de crianza resulta el democrático el más aconsejable, no así el más fácil de mantener, pues predomina la horizontalidad en las relaciones entre padres e hijos sin que los primeros pierdan su rol de decisores y responsables de la autoridad.

En Cuba la protección legal de la niñez y la adolescencia es un hecho cierto en el sistema de leyes cubanas, que está íntimamente vinculado a los postulados constitucionales que rigen las instituciones jurídicas de la familia, en la comunidad y la sociedad civil y por tanto condiciona el ejercicio pleno de sus derechos.

Los principales retos para perfeccionar este Sistema de Disposiciones Jurídicas, le corresponden al Código de Familia que debe ser oportunamente modificado en aras de regular expresamente los derechos que tendrían los niños sujetos al régimen de patria potestad, y entre los que pudiéramos mencionar:

16. Ídem. 
-El derecho a ser protegido de toda forma de violencia física y psíquica, explotación, abuso sexual, descuido o trato negligente.

-El derecho de los infantes a participar activamente en la vida familiar y social, a que sus opiniones sean escuchadas, a defender sus criterios y a recibir argumentos que puedan convencerlos.

Y además establecer la posibilidad de deferir la patria potestad a favor de los abuelos u otros familiares antes situaciones prácticas que así lo justifiquen.

\section{REFERENCIAS}

- Carballo Vargas, Sonia. "Desarrollo Humano y Aprendizaje: Prácticas de crianza de las madres Jefas de Hogar”. Revista Electrónica Actualidades Investigativas en la Educación. Universidad de Costa Rica. Vol. 6. No. 2, mayo-agosto (2006): 2. Acceso el 13 de noviembre de 2019 desde http://www.un.org/es/events/childrenday/pdf/derechos.pdf

- Cartilla 8 "Crianza Positiva" del Instituto Colombiano de bienestar familiar-ICBF (2015): 2-3. Acceso 13 de noviembre de 2019 desde https:/www.aldeasinfantiles.org.co/getattachment/que-es-aldeas/Prensa/noticias/Acompanarte-elarte-de-acompanar/CARTILLA-8-CRIANZA-POSITIVA.pdf

- Constitución de la República de Cuba. Gaceta Oficial No. 5, Extraordinaria de 10 de abril de 2019.

- Código de Familia. Ley No 1289 de 14 de febrero de 1975. (anotado y concordado) de la República de Cuba, Divulgación del Ministerio de Justicia, abril de 1987.

- Código de la Niñez y la Juventud. Ley No.16 de 28 de junio de 1978.

- Código Penal. Ley No. 62 de 1987, actualizado, Colección Jurídica, Ministerio de Justicia, La Habana. Gaceta Oficial de la República de Cuba, Extraordinaria No.1, de 15 de marzo de 1999.

- ¿Cómo llevar a cabo una crianza positiva? Acceso 16 de marzo de 2019 desde https://www.bekiapadres.com/articulos/como-llevar-cabo-crianza-positiva/

- Domínguez Cruz, Ana María. “¿Cómo ser mejores madres y padres?”. Periódico Juventud Rebelde, Diario de la Juventud Cubana, martes 29 de septiembre 2015.

- Decreto -Ley No. 64 de 1982 "Sobre el Sistema de Atención a Menores con trastornos de Conducta”.

- Decreto- Ley No. 76 de 1984 crea la Red Nacional de Círculos Infantiles Mixtos y Hogares de Menores sin Amparo Familiar, para la protección de los niños en desventaja social. 
- Horno Goicoechea, Pepa. "Atención a los niños y las niñas víctimas de la violencia de género".

Revista Intervención Psicosocial Vol.15, No.3, (2006): pp. 307-316. Acceso 28 de octubre de 2019 desde http://scielo.isciii.es/pdf/inter/v15n3/v15n3a05.pdf https://doi.org/10.4321/S1132-05592006000300005

- $\quad$ Ley 51/1985 de 15 de julio, Del Registro del Estado Civil, publicación del MINJUS, La Habana,1998 y su Reglamento contenido en la Resolución 157/1985 de 25 de diciembre del Ministro de Justicia, publicación del MINJUS, La Habana, 1998.

- Pérez Sáez, Dora. "Nuevo Código de Familia favorece a los niños". Periódico Juventud Rebelde, Diario de la Juventud Cubana, de 23 de marzo de 2019, desde https://www.juventudrebelde.cu/cuba/.../el-nuevo-código-de-familia-favorecerá-a-los-niños

- "Principios rectores de la Convención sobre los Derechos del Niño: una explicación fácil”. Acceso 3 de abril de 2019 desde https://www.crin.org/es/biblioteca/publicaciones/principios-rectores-de-la-convencion-sobre-losderechos-del-nino-una

- Steven, Dowshen. "Nueve pasos para una crianza más eficaz para padres". Acceso 8 de marzo de 2020 desde https:// kidshealth.org

- $\quad$ Sistema de leyes que protegen la nińez y a los adolescentes en Cuba. Acceso 13 de febrero de 2019 desde http://civica.cubaeduca.cu/sistema-de-leyes-que-protegen-a-la-ninez-y-a-los-adolescentes-en-cuba \#:- :text=\%C2\%BFConoces\%20cu\%C3\%A11\%20es\%20el\%20sistema,los\%20adolescentes\% 20en\%20nuestro\%20pa\%C3\%ADs\%3F\&text=En\%20el\%20sistema\%20de\%20disposiciones, regulado\%20en\%20la\%20Ley\%20No.

- Roldán, María José. “La crianza positiva de los hijos-Guía infantil”. Acceso 22 de enero 2019 desde https://www.guiainfantil.com

RECIBIDO: 09/09/2020

APROBADO: $15 / 10 / 2020$ 